\title{
Diode laser trabeculoplasty (DLT) for primary open-angle glaucoma and ocular hypertension
}

\author{
Dominic McHugh, John Marshall, Timothy J ffytche, Peter AM Hamilton, Anthony Raven
}

\begin{abstract}
A pilot study has been carried out to examine the efficacy of diode laser trabeculoplasty in the treatment of primary open-angle glaucoma and ocular hypertension. The device used was portable and could be attached to a standard slit-lamp microscope. Powers of 0.8-1.2 W were used, with a spot size of $100 \mu \mathrm{m}$ and a pulse of 0.20 second. Laser trabeculoplasty carried out on 20 eyes for glaucoma resulted in a mean ocular hypotensive effect of $10.2 \mathrm{mmHG}$ at two weeks after treatment and of $9.55 \mathrm{mmHg}$ at 6 months. It is concluded that diode laser trabeculoplasty is an effective mode of treatment for eyes with open-angle glaucoma or with ocular hypertension.
\end{abstract}

Primary open-angle glaucoma (POAG) is a common, potentially blinding ocular condition. It is characterised by a raised intraocular pressure (IOP), with secondary damage to the optic nerve, and consequent loss of visual field. Treatment is directed towards lowering the IOP, and the first line of treatment consists in topical medication with an ocular hypotensive, for example timolol, often in conjunction with oral therapy such as acetazolamide. However, the response may be insufficient by pharmacological methods alone.

Laser trabeculoplasty (LTP) for the treatment of uncontrolled glaucoma was first described by Wise and Witter in 1979. ${ }^{1}$ It has since become an established treatment, with several studies demonstrating its effectiveness. ${ }^{23}$ It is a technique whereby a number of laser burns are applied to the trabecular meshwork of the angle of the anterior chamber of the eye. LTP is usually performed with an argon laser, which emits blue-green radiation (wavelength 488-514.5 nm), though a similar ocular hypotensive action has been demonstrated with krypton red $(647 \mathrm{~nm})^{4}$ and neodymium-YAG $(1064 \mathrm{~nm})$ laser radiation. ${ }^{5}$

In the present study a portable semiconductor diode laser, emitting at $810 \mathrm{~nm}$, was used to examine the clinical effects of near-infrared irradiation of the trabecular meshwork. The purpose of this study was to evaluate the ocular hypotensive action of diode laser trabeculoplasty (DLT) in the treatment of patients with primary open-angle glaucoma. In this preliminary investigation randomised comparison with argon laser trabeculoplasty was not performed.

\section{Materials and methods}

INSTRUMENTATION

A diode laser was used in the study which could be attached to a standard Haag-Streit slit-lamp. The maximum available power of the infrared treatment beam was $1.4 \mathrm{~W}$. The aiming beam was provided by a low-power, red-emitting laser diode. When it was not in use the laser could be stored in a carrying case, which enabled treatments to be carried out at two centres with the same instrument. A more detailed description of the laser is provided elsewhere. ${ }^{6}$

\section{INCLUSION CRITERIA FOR PATIENTS}

Patients were recruited to the study from general clinics at Moorfields Eye Hospital and from the Department of Ophthalmology at St Thomas's Hospital. The following inclusion criteria were established for the study:

(a) The patient should have primary openangle glaucoma for which medical therapy had failed to satisfactorily control intraocular pressures. Because the emphasis of the study was directed at the effect on IOP, patients with poorly controlled ocular hypertension but no visual field abnormalities were also admitted to the study. Patients with forms of secondary glaucoma - for example, resulting from uveitis, or aphakia were excluded.

(b) The pretreatment IOP should be $22 \mathrm{mmHg}$ or greater.

(c) The patient should be able to provide informed consent to participate in the study and be willing and able to attend for follow-up visits.

(d) There should be no corneal opacities, such as band keratopathy, that precluded adequate visualisation of the trabecular meshwork.

(e) No previous photocoagulation or operative surgery should have been performed on the trabecular meshwork.

\section{PATIENT EVALUATION}

Pretreatment evaluation of the patient consisted of:

(a) Corrected visual acuity with an illuminated Snellen chart at 6 metres.

(b) Biomicroscopic examination of the anterior segment, including: Intraocular pressure measurement with a Goldmann applanation tonometer. All measurements of IOP were performed in the morning. Gonioscopy using a Goldmann 3-mirror contact lens. The width of the anterior chamber angle was estimated on a scale of $0-4$ on the Shaffer grading. Funduscopy included an examination of the optic discs, with an estimation of the cup:disc ratio and the degree and extent of pallor. Colour stereo photographs of the optic discs were taken in those eyes without pharmacologically induced miosis. 
TREATMENT PROTOCOL

All patients were given a full explanation of the treatment and possible side effects before signing consent forms. Included in the discussion was a statement that some discomfort may be associated with the treatment. Therapy was carried out on a separate day from that of the pretreatment assessment. No adjustment was made to the patient's glaucoma medication prior to laser therapy.

Twenty eyes in 13 patients were treated. Sixteen eyes had chronic open-angle glaucoma, three eyes had ocular hypertension (with raised intraocular pressures but normal visual fields), and one eye had pseudoexfoliation glaucoma. Eight patients were female and five male. Two patients were black, the others were Caucasian. The mean age was 65 (range 57-76).

After instillation of a topical anaesthetic (amethocaine 1\%), a Goldmann or Ritch contact lens was applied to the eye in order to allow visualisation of the anterior chamber angle. All treatments were performed throughout the study by one clinician (DM), who had prior experience in the use of lasers for the management of glaucoma. The pattern of treatment consisted in applying approximately 50 burns to $180^{\circ}$ of the trabecular meshwork, The laser spot size was $100 \mu \mathrm{m}$ and the pulse duration was $0 \cdot 20$ second. The target area was the pigmented portion of the trabecular meshwork. The power was adjusted to produce a mild blanching of the trabecular meshwork.

The intraocular pressures were measured two to three hours after therapy. A transient rise in pressure would be treated with a drop of timolol $0.5 \%$. The patients were asked to continue with their current glaucoma medication.

\section{POST-TREATMENT REVIEW}

The patients were reviewed at two weeks, six weeks, and 12 weeks after treatment and at twoto three-month intervals thereafter. At each visit the ocular examination included measurement of visual acuity, anterior segment examination, intraocular pressures and funduscopy, including examination of the optic discs.

If a satisfactory ocular hypotensive effect was achieved, with the IOP less than $22 \mathrm{mmHG}$, a reduction of the patient's ocular medication was considered.

An unsatisfactory response to treatment was considered to be failure of the intraocular pressure to fall to less than $22 \mathrm{mmHg}$ at six weeks after laser therapy. In this case laser trabeculoplasty was repeated to the other $180^{\circ}$ of the angle. Continued failure to control the IOP would lead to consideration of operative intervention.

Table 1 Changes in intraocular pressure induced by diode laser trabeculoplasty (DLT)

\begin{tabular}{lc}
\hline Mean pretreatment IOP (mmHg) & $28 \cdot 30(\mathrm{SD}=3 \cdot 63)(\mathrm{n}=20)$ \\
Mean IOP at 2 weeks following DLT & $18 \cdot 10(\mathrm{SD}=2 \cdot 53)(\mathrm{n}=20)$ \\
Mean reduction in IOP at 2 weeks & $10 \cdot 20(\mathrm{SD}=3 \cdot 25)(\mathrm{p}=<0 \cdot 001)$ \\
Mean IOP at 6 weeks following DLT & $19 \cdot 10(\mathrm{SD}=2 \cdot 88)(\mathrm{n}=20)$ \\
Mean reduction in IOP at 6 weeks & $9 \cdot 20(\mathrm{SD}=4 \cdot 48)(\mathrm{p}=<0 \cdot 001)$ \\
Mean IOP at 3 months following DLT & $19 \cdot 00(\mathrm{SD}=3 \cdot 63)(\mathrm{n}=20)$ \\
Mean reduction in IOP at 3 months & $9 \cdot 30(\mathrm{SD}=4 \cdot 70)(\mathrm{p}=<0.001)$ \\
Mean IOP at 6 months following DLT & $18 \cdot 75(\mathrm{SD}=3 \cdot 11)(\mathrm{n}=20)$ \\
Mean reduction in IOP at 6 months & $9 \cdot 55(\mathrm{SD}=3 \cdot 36)(\mathrm{p}=<0.001)$ \\
\hline
\end{tabular}

Table 2 Mean reduction in IOP following diode laser trabeculoplasty in comparison with treatment with other lasers

\begin{tabular}{lll}
\hline & $\begin{array}{l}\text { Reduction in IOP } \\
\text { at 2-4 weeks } \\
(m m H g)\end{array}$ & $\begin{array}{l}\text { Reduction in IOP } \\
\text { at 4-6 weeks } \\
(m m H g)\end{array}$ \\
\hline Laser treatment & $10 \cdot 2(\mathrm{n}=20)$ & $9 \cdot 20(\mathrm{n}=20)$ \\
Argon & $6 \cdot 9(\mathrm{n}=26)$ & $9 \cdot 7(\mathrm{n}=34)$ \\
Argon† & $4 \cdot 0(\mathrm{n}=61)$ & $5 \cdot 5(\mathrm{n}=61)$ \\
cw.Nd-YAG & $6 \cdot 2(\mathrm{n}=33)$ & $6 \cdot 3(\mathrm{n}=33)$ \\
\hline
\end{tabular}

^From Schwartz et al.

tFrom Fink et al ${ }^{16}$

†From Belgrado et al.

\section{Results}

USE OF INSTRUMENTS

No problems were encountered with the slitlamp mountable version of the diode laser in conjunction with a Goldmann 3-mirror or a Ritch lens. Visualisation of the angle of the anterior chamber was not difficult, and the red aiming beam was easily focused on the trabecular meshwork.

\section{LASER BURNS}

The tissue reaction that was commonly observed following laser exposure was a blanching of the pigmented portion of the trabecular meshwork. This generally occurred at power settings of between 800 and $1200 \mathrm{~mW}$. In eyes with poor trabecular pigmentation this response was more difficult to identify. Formation of gas bubbles at the site of laser irradiation was not observed.

\section{CLINICAL RESULTS}

The mean period of review was eight months (range 6-12 months). Seven patients had bilateral DLT, and six had treatment to one eye only. Sixteen eyes had one treatment with the diode laser, and four eyes had two treatments.

Intraocular pressures. The mean pretreatment intraocular pressure was $28.3 \mathrm{mmHg}$ (range 22-36). The mean IOP two weeks after laser trabeculoplasty was $18 \cdot 1 \mathrm{mmHg}$ (range 14-22). It was noted that in three of the six patients who had treatment to one eye the intraocular pressure was lower in the untreated eye than before treatment (by 3, 4, and $8 \mathrm{mmHg}$ ). In the remaining untreated eyes there was no change in intraocular pressure.

Table 1 shows that the ocular hypotensive effect of laser therapy was maintained in the treatment group over a period of six months following treatment. The paired $t$ test, which determines the difference between matched samples, was used to assess changes in IOP from the baseline pretreatment level. The results indicate that therapy was associated with a significant reduction in intraocular pressure, which was maintained over the period of review. Table 2 allows historical comparison with results from previous studies which utilised other laser wavelengths for trabecular irradiation. Fig 1 and 2 illustrate the IOPs before and at (Fig 1) two weeks and (Fig 2) six months following treatment in each of the eyes. Of the five eyes which have been reviewed for longer than six months four have had IOPs of less than $22 \mathrm{mmHg}$. One 


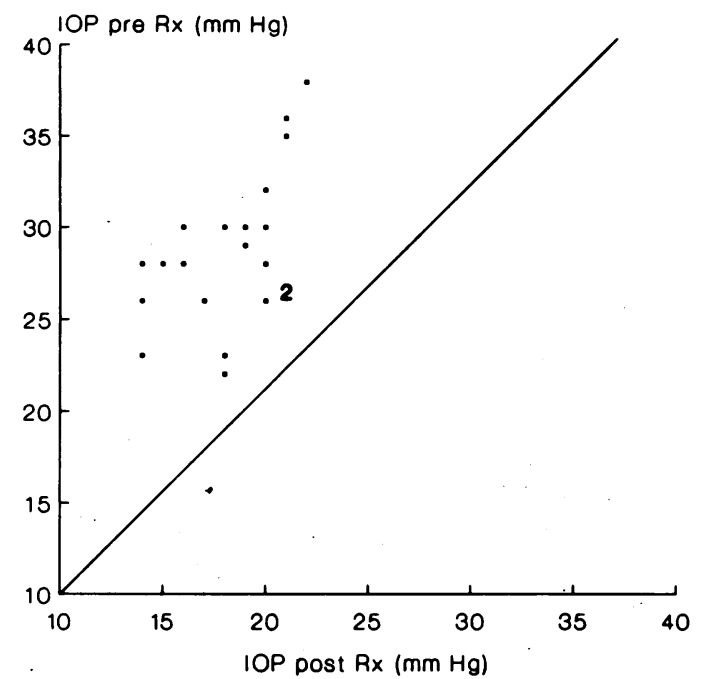

Figure 1 Intraocular pressures before and at two weeks after diode laser trabeculoplasty (20 eyes). Treatment was associated with a reduction in pressure ranging from 4 to $14 \mathrm{mmHg} . \mathrm{Rx}=$ treatment.

eye had a measured pressure of $25 \mathrm{mmHg}$ at 10 months and evidence of further loss of visual field. It was therefore decided to perform a trabeculectomy on this patient.

In this small series no particular factors were identified which would tend to reduce the success of treatment. One of the black patients treated had an initial reduction in IOP from 28 , $22 \mathrm{mmHg}$, to $16,18 \mathrm{mmHg}$ at two weeks. At six weeks and 24 weeks following treatment the pressures had been 20,18 , and $21,19 \mathrm{mmHg}$. Any further increase of IOP in the right eye will require a second trabeculoplasty to be carried out.

Two patients had their hypotensive medication adjusted. One patient had returned four weeks after treatment to both eyes. She had suffered a myocardial infarction and her glaucoma medication of acetazolamide $250 \mathrm{mg}$ four times a day, pilocarpine $4 \%$ four times a day, and timolol $0.25 \%$ twice a day had been stopped. On examination her intraocular pressures were 36

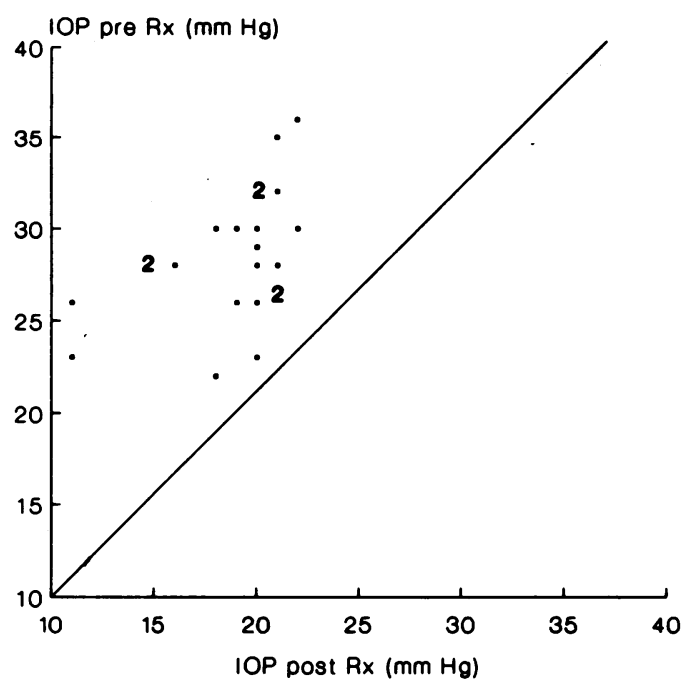

Figure 2 Intraocular pressures before and at six months after diode laser trabeculoplasty (20 eyes). The ocular hypotensive effect of laser treatment appears to have been maintained. $R x=$ treatment. and $38 \mathrm{mmHg}$. DLT was repeated to each eye and the patient was started on pilocarpine $4 \%$ eye drops. Two weeks later her intraocular pressures were $22 \mathrm{mmHg}$ in each eye, and six weeks after therapy they were $21 \mathrm{mmHg}$ bilaterally. The second patient had pretreatment pressures of $28 \mathrm{mmHg}$ bilaterally and was being treated with adrenaline $1 \%$ eye drops and timolol $0.5 \%$ eye drops twice daily to each eye, in addition to acetazolamide $250 \mathrm{mg}$ four times a day. Three months after treatment his IOPs were 15 and $14 \mathrm{mmHg}$. The adrenaline drops were stopped and the dose of acetazolamide was reduced to $250 \mathrm{mg}$ twice a day. At five months the pressures were well controlled at $16 \mathrm{mmHg}$ in each eye.

Visual acuity. There was no change in visual acuity in any of the patients during the period of review.

Optic discs. During the relatively brief followup there was no change in the appearances of the optic discs in the eyes treated.

Complications of therapy. In four of the eyes treated a mild cellular reaction in the anterior chamber was noted two hours after therapy. This was not considered to require anti-inflammatory treatment.

In no eyes was there an acute increase in IOP over pretreatment levels. In six eyes there was a small decrease in IOP. This was attributed to the effect of the contact lens pressing on the eye and resulting in expulsion of aqueous.

Several patients noted a mild 'pricking' sensation during therapy, but no patients complained of excessive discomfort.

\section{Discussion}

It is difficult to draw clear-cut conclusions from this study owing to the small size of the treatment group and the limited period of review. Historical comparisons may be made with results from previous studies in order to gain some indications of the efficacy of diode laser trabeculoplasty compared with other forms of laser treatment.

Although LTP is usually performed with an argon laser, ${ }^{1-3}$ a similar ocular hypotensive action has been demonstrated with lasers emitting at other wavelengths. Spurney and Lederer $^{4}$ reported performing laser trabeculoplasty with a krypton red (647 $\mathrm{nm}$ ) or krypton yellow laser. A mean reduction in IOP of $9.6 \mathrm{mmHg}$ followed krypton red therapy and $7.0 \mathrm{mmHg}$ after krypton yellow treatment.

A study in which the effects of LTP were compared using a cw neodymium-YAG (1064 nm) laser and an argon blue-green laser showed similar therapeutic effects, though the short pulse duration of the YAG laser $(20 \mathrm{~ms})$ rendered the results not strictly comparable. ${ }^{5}$

Table 2 coinpares the results of the present study with those reported in previous trials at up to two months after treatment. It shows that the early therapeutic effects of LTP are similar and that they do not appear to depend on the wavelength of the laser employed, though there is some indication that diode infrared irradiation tends to effect a greater early reduction in pressure than argon blue laser treatment.

The precise mechanism for the pressure 
lowering effect of LTP is unknown. One theory suggests that photocoagulation results in contraction of trabecular fibres, which opens Schlemm's canal by drawing the attached trabecular fibres inward. ${ }^{7}$ Another proposes that laser irradiation causes degenerative decay of the trabecular fibres, with a secondary widening of the intertrabecular spaces. ${ }^{8}$ Whatever the mechanism of action, it is agreed that the beneficial effect of LTP is to cause a decrease in resistance to aqueous outflow ${ }^{9}$ and an improvement in aqueous outflow facility. ${ }^{210}$ There is also evidence to suggest a more complex biological response to irradiation, with an increase in trabecular cell division, which may also influence the outflow facility. ${ }^{11}$

The observation of a comparable hypotensive effect of diode laser trabeculoplasty with treatment by lasers of other wavelengths implies a similar mode of action. The deeper penetration of infrared into the relatively poorly pigmented trabecular meshwork than with argon blue has been observed in a recent histological study. ${ }^{12}$ Possibly this results in a more marked effect on intraocular pressure, either through a greater degree of opening of the trabecular spaces or in promoting more widespread division of those trabecular cells which may play an active role of aqueous outflow. ${ }^{13}$ The validity of this speculation awaits the results of further investigations.

The desired visible end point of a trabecular exposure was a mild blanching of the pigmented portion of the trabecular meshwork. This was based on the responses noted during histopathological studies. ${ }^{12}$ Gas bubble formation was not a feature of any reaction. This may be related to the characteristics of the tissue response to infrared irradiation, in that the dissipation of energy over a greater path length results in a lower posibility of explosive tissue disruption. Alternatively, it may be due to the powers selected being below the threshold required for formation of gas bubbles.

It is a feature of the treatment protocol of several series that gas bubble formation should be caused as part of the end point. ${ }^{210}$ The rationale for this has never been satisfactorily explained. Indeed there is some evidence that it has an adverse effect on the eye. It is recognised that LTP is often accompanied by a transient rise in intraocular pressure. ${ }^{14}$ This phenomenon may put any eye which is already compromised at risk of further loss of visual field. ${ }^{15}$

The present study with the diode laser found no acute rise in IOP following therapy in any of the eyes treated, even in those eyes with deeply pigmented trabeculae - for example, in black patients. As the desired reaction was to produce a mild blanching of the trabecular meshwork with each burn and bubble formation was not a feature of the exposures, it seems clear that the lesions were only just above threshold in character. There would then be a reduced likelihood of excessive damage to trabecular fibres and of obstruction to aqueous outflow. The mildness of anterior segment inflammation following therapy may also be related to the powers employed in treatment.

Without a detailed knowledge of the mechanisms underlying laser trabeculoplasty it is difficult to optimise the procedure; but clearly the beneficial effects of laser trabeculoplasty do not depend on excessive irradiation of the trabeclar meshwork. One study treated patients with argon LTP, with one group being treated with 100 burns at $1 \mathrm{~W}$, and the other group being treated with 65 burns at $850 \mathrm{~mW}$. No significant difference was found in the rate of success for each group. ${ }^{16}$ Tissue culture experiments have been performed in which cat trabecular meshwork was irradiated with an argon laser at power settings of $300 \mathrm{~mW}$ and at $1000 \mathrm{~mW}$, with no differences being found in the rate of induced cell division between the two groups. ${ }^{17}$

The powers used in the present study were of the order of 1 watt and therefore similar to those used with argon LTP. However, the lower absorption of infrared $(810 \mathrm{~nm})$ within melanin compared with that of argon blue-green radiation (488-514.5 nm) $)^{18}$ implies less energy deposition with diode irradiation at comparable power levels. This should lead to less tissue damage with diode exposures, but clearly a similar clinical response was observed.

It was interesting that in several patients a reduction in pressure was noted in the contralateral, untreated eye. This was described by Fink et al, ${ }^{19}$ in their series of 34 eyes. They found an average decrease of $4.2 \mathrm{mmHg}$ in the untreated eyes compared with $9.9 \mathrm{mmHg}$ in the treated eyes. This raises the possibility of the influence of neurogenic factors or systemically disseminated chemical mediators.

The limited review period of the present series does not allow any conclusions to be drawn regarding the longer term efficacy of diode laser trabeculoplasty. Previous studies of argon LTP report a maximum pressure lowering effect occurring between two months ${ }^{20}$ and 6-9 months after treatment. ${ }^{16}$

Previous studies have demonstrated the clear cut ergonomic advantages of diode lasers over current ion laser systems in relation to their compactness and greater reliability. ${ }^{621}$ The effectiveness of laser trabeculoplasty (as with other forms of treatment) in slowing the sight threatening consequences of glaucoma has still not been fully established. The present study has, however, shown that infrared diode laser trabeculoplasty is effective in the control of intraocular pressure and that it is a treatment with minimal ocular side effects. It thus represents a sound adjunct to, or replacement of, pharmacological therapy and a means of deferring drainage surgery, with its attendant risk of damage to sight. ${ }^{22}$

We thank Keeler Ltd for providing the diode laser that was used in the study.

J D A McHugh was supported by a grant from the Allerton Vision Research Trust.

1 Wise JB, Witter SL. Argon laser therapy for open angle glaucoma: a pilot study. Arch Ophthalmol 1979; 97: 319-22.

2 Schwartz AL, Whitten ME, Bleimen B, et al. Argon laser trabecular surgery in uncontrolled phakic open-angle glaucoma. Ophthalmology 1981; 88: 203-12.

3 Pollack IP, Robin AL, Sax H. The effect of argon lase trabeculoplasty under medical control of primary open angle trabeculoplasty under medical control of prim

4 Spurny RC, Lederer CM Jr. Krypton laser trabeculoplasty: a clinical report. Arch Ophthalmol 1984; 102: 1626-8. 
5 Belgrado G, Brihaye-Van Geertruyden, Herzeel R. Comparison of argon and cw.Nd:YAG laser trabeculoplasty. Clinical results. In: Marshall J. ed. Laser technology in ophthalmology. Berkeley: Kugler, Ghedini, 1988: 45-8.

6 McHugh JDA, Marshall J, ffytche TJ, Hamilton AM, Raven A, Keeler CR. Initial clinical experience using a diode laser in the treatment of retinal vascular disease. Eye 1989; 3 : 516-27.

7 Weber PA, Davidorf FH, McDonald C. Scanning electron microscopy of argon laser trabeculoplasty. Ophthalmic Forum 1983; 1: 26-9.

8 Van der Zypen E, Fankhouser F. Ultrastructural changes of the trabecular meshwork of the monkey (Macaca speciosa) the trabecular meshwork of the monkey (Macaca speciosa)

9 Brubaker RF, Liesegang TJ. Effect of trabecular photocoagulation on the aqueous humor: dynamics of the human eye. Am F Ophthalmol 1983; 96: 139-47.

10 Wilensky JT, Jampol LM. Laser therapy for open-angle glaucoma. Ophthalmology 1981; 88: 213-7.

11 Bylsma SS, Samples JR, Acott TS, Van Budkirk EM. Repopulation of the trabecular meshwork after argon laser trabeculoplasty. Invest Ophthalmol Vis Sci 1988; 29 (suppl): 129.

12 McHugh JDA, Marshall J, ffytche TJ, Hamilton AM, Raven A. Diode laser trabeculoplasty (DLT) for chronic simple glaucoma. Invest Ophthalmol Vis Sci 1990; 31 (suppl): 340.

13 Bylsma SS, Samples JR, Acott TS, Van Buskirk EM. Trabecular cell division following argon laser trabeculoplasty.

14 Weinreb RN, Ruderman J, Juster R, Sweig K. Immediate intraocular pressure response to argon laser trabeculoplasty. Am f Ophthalmol 1983; 95: 279-86.

15 Levene R. Major early complications of argon laser trabeculoplasty. Ophthalmic Surg 1983; 14: 947-53.

16 Fink AI, Jordan AJ, Lao PN, Fong DA. Therapeutic limitations of argon laser trabeculoplasty. Br $\mathcal{F}$ Ophthalmol 1988; 72: 263-9.

17 Bylsma SS, Pirouzkar B, Acott TS, Van Buskirk EM, Samples JR. In-vivo cell division after argon laser trabeculoplasty. Invest Ophthalmol Vis Sci 1989; 30 (suppl): 280.

18 Gabel VP, Birngruber R, Hillenkamp F. Individuelle Unterscheide der Lichtabsorption am Augenhintergrund in sichtbaren und infraroten Spectralbereich. Ber Dtsch Ophthalmol Ges 1977; 47: 418-21.

19 Fink AI, Jordan AJ, Bunke A, Fong DA. Argon laser trabeculoplasty as an alternative to carbonic anhydrase trabeculoplasty as an alternative to carbonic anh
therapy. Trans Ophthalmol Soc UK 1982; 102: 125-8.

20 Schwartz AL, Love DC, Schwartz MA. Long-term follow-up of argon laser trabeculoplasty for uncontrolled open-angle glaucoma. Arch Ophthalmol 1985; 103: 1482-4.

21 McHugh JDA, Marshall J, Capon M, et al. Transpupillary photocoagulation in the eyes of rabbit and human using a diode laser. Lasers Light Ophthalmol 1988; 2: 125-43.

22 Watson PG, Grierson I. The place of trabeculectomy in the treatment of glaucoma. Ophthalmology 1981; 88: 175-96. 